\title{
A DUALITY PRINCIPLE
}

\author{
WOLFGANG SANDER
}

\begin{abstract}
With the aid of the Baire category theory we prove an extension of Erdös' well-known duality principle concerning sets of Lebesgue measure zero and sets of first category.
\end{abstract}

Assuming the continuum hypothesis, Sierpinski [9] proved the existence of a bijective function $f: \mathbf{R} \rightarrow \mathbf{R}$, such that $A$ is of first category iff $f(A)$ has Lebesgue measure zero. By a modification of Sierpinski's proof, Erdös [2] showed that the function $f$ in Sierpiński's result can be chosen such that $f=f^{-1}$. The importance of these results is that they allow one to state a well-known duality principle (see [6]). Using Morgan's abstract Baire category theory (cf. [3-5]), Cholewa [1] generalized Sierpiński's theorem, but an analogous generalization of Erdös' theorem is not known.

The aim of this paper is to give a short proof of a new extension of Erdös' result. In this note we assume, that $X$ is a topological group, which is a complete, separable, metric space without isolated points; moreover we suppose that the reader is familiar with Morgan's theory.

THEOREM 1. Let $c=\omega_{1}$. If $\mathcal{C}$ and $\mathscr{Q}$ are nonequivalent $\mathfrak{B}$-families [4] and $\subseteq$-families on $X$, satisfying c.c.c. (countable chain condition), then there is a bijective function $f$ : $X \rightarrow X$ such that $f=f^{-1}$ and such that $A \in \mathcal{C}_{\mathrm{I}}$ iff $f(A) \in \mathscr{D}_{\mathrm{I}}$.

Proof. The properties of $X$ imply that $|X|=c$, since otherwise

$$
X=\cup\{\{x\}: x \in X\}
$$

would be of first category. We note that $\bigodot_{\mathrm{I}}$ is a $\sigma$-ideal such that $X=\cup \mathcal{C}_{\mathrm{I}}$, since $\{x\} \in \mathcal{C}_{\mathrm{I}}$ for all $x \in X[4$, Theorem 6]. Now let

$$
\mathrm{g}:=\left\{A \in \mathfrak{\ell}_{\delta \sigma} \cap \mathcal{e}_{\mathrm{I}}:|A|>\boldsymbol{\aleph}_{0}\right\},
$$

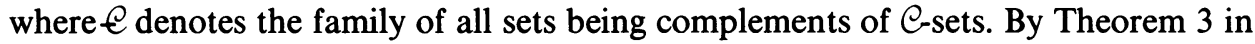
[5] and by Corollary 10 in [4] each $\bigodot_{\mathrm{I}}$-set is contained in some $g$-set. Since $\mathcal{C}$ consists of perfect sets, we get $|\mathcal{C}| \leqslant c$ and thus $|\mathfrak{g}| \leqslant c$. Moreover, the complement of a $\mathcal{C}_{\mathrm{I}}$-set lies in $\mathfrak{B}(\mathcal{C}) \cap \mathcal{C}_{\mathrm{II}}$ and by Corollary 15 in [4], it contains a nonempty

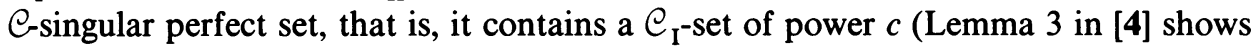
that, without loss of generality, we can assume, that $X \in \mathcal{C} \subset \mathcal{C}_{\text {II }}$ ).

Received by the editors February 15, 1981.

. 1980 Mathematics Subject Classification. Primary 54E50, 22A10; Secondary 28A05.

Key words and phrases. Sets of first category, sets of Lebesgue measure zero, Baire category from an abstract viewpoint, complete metric spaces, duality principle. 
Thus $C_{I}$ fulfills the properties (a)-(d) of Theorem 19.5 in [6]. It is clear, that the same fact is true for $\mathscr{D}_{\mathrm{I}}$. Since $C_{\text {and }} \mathscr{D}$ are nonequivalent, it follows from Theorem 2 in [8], that $X$ is the disjoint union of a $C_{\mathrm{I}}$-set and a $\mathscr{D}_{\mathrm{I}}$-set. Thus, by Theorem 19.6 in [6], the proof is finished.

The classical result of Erdös can be obtained from Theorem 1 by setting $\mathcal{C}:=\{\{x \in \mathbf{R}:|x-y| \leqslant 1 / n\}: y \in \mathbf{R}, n \in \mathbf{N}\}$ and $\mathscr{D}:=\{A \subset \mathbf{R}: A$ is closed, $\forall x \in A \forall U \in \tau x: \mu(U \cap A)>0\}$ ( $\tau x$ consists of all open subsets of $\mathbf{R}$ containing $x$ and $\mu$ denotes the Borel measure in $\mathbf{R}$ ). It follows, that $\mathcal{C}$ and $\mathscr{D}$ are nonequivalent $\mathfrak{B}$ and $\subseteq$-families (see [4 and 8]). Since the members of $\mathcal{C}$ and $\mathscr{D}$ have positive measure, $\mathcal{C}$ and $\mathscr{D}$ also satisfy c.c.c. $[7$, p. 123].

\section{REFERENCES}

1. P. Cholewa, Sierpinski-Erdös duality principle in the abstract Baire-category theory, manuscript.

2. P. Erdös, Some remarks on set theory, Ann. of Math. (2) 44 (1943), 643-646.

3. J. C. Morgan II, On translation invariant families of sets, Colloq. Math. 34 (1975), 63-68.

4. __ The absolute Baire property, Pacific J. Math. 65 (1976), 421-436.

5.

6. J. C. Oxtoby, Measure and category, Springer-Verlag, Berlin-Heidelberg-New York, 1971.

7. C. A. Rogers, Hausdorff measures, Cambridge Univ. Press, London and New York, 1970.

8. W. Sander, A decomposition theorem, Proc. Amer. Math. Soc. 83 (1981), 553-554.

9. W. Sierpiński, Sur la dualité entre la première catégorie et la mesure nulle, Fund. Math. 1 (1920), $105-111$.

Institut C fur Mathematik, Technische Universität Braunschweig, Pockelsstr. 14, D 3300 BraunschWeig, West Germany 